\title{
Research on Ferroelectret Based Ultrasound Transducers
}

\author{
Alexander Sutor ${ }^{1,2}$, Stefan J. Rupitsch ${ }^{1}$, Reinhard Lerch ${ }^{1}$ \\ ${ }^{1}$ Chair of Sensor Technology, University of Erlangen-Nuremberg, Germany, Paul-Gordan-Str. 3/5, 91052 \\ Erlangen \\ ${ }^{2}$ sutor@Ise.eei.uni-erlangen.de
}

\begin{abstract}
Ferroelectrets are excellent materials for electroacoustic transducers. The materials offer both a rather large bandwidth and a high piezoelectric strain constant. Due to their cellular structure, the materials are flexible and exhibit good matching to air. Therefore, these polymers are appropriate for many sound as well as ultrasound transducer applications. Our research is concentrated on the simulation based design and characterization of ultrasound transducers. We fabricate single element and array transducers based on ferroelectret materials, namely the so-called EMFi (Electro-Mechanical-Film) material. In order to show the applicability of ferroelectret materials for ultrasound transducers, we have developed different applications for air and water. Here, we present a robust sensor array consisting of 16 single elements $(4 \times 4)$. With the aid of this sensor array, cavitation effects in ultrasonic cleaning systems can be investigated on the specimen's surface, which is not possible with common ultrasound sensors, e.g., hydrophones.
\end{abstract}

\section{Introduction}

Ultrasound is widely used in industrial applications and in NDE (Nondestructive Destructive Evaluation) as well as medical diagnostics and therapy [1-4]. In the majority of cases, piezoceramic materials are utilized for ultrasound emitters and receivers. However, those materials exhibit several drawbacks, which limit the performance of the transducers. For instance, the high acoustic impedance of piezoceramic materials requires additional acoustic matching layers on top of the transducer surface for air and liquid applications [1,2]. In addition, piezoceramics are brittle and can, therefore, hardly be fixed to curved surfaces. Hence, various applications ask for alternative transducer materials allowing high bandwidth devices.

About a decay ago such an alternative transducer material was invented and first transducers based on it were presented by Sessler et al. [5] and Paajanen et al. [6]. This material is a cellular structured ferroelectret polymer. According to the piezoelectric properties of the material after corona discharge, it can be used for transducers operating as emitter or receiver of ultrasound waves $[1,7]$. Due to their cellular structure, ferroelectret materials offer an excellent impedance matching to air and, consequently, a rather large bandwidth. That are the reasons why these materials are utilized for transducer applications in the audible sound range (see, e.g., [8]) as well as recently in the ultrasound range [9-11]. The so-called EMFi (Electro-Mechanical-Film) material is currently a well-established representative of the ferroelectret materials. It is flexible, cheap and provides a high piezoelectric strain constant [12-15].
The main focus of our research lies on the development of electroacoustic transducers for ultrasound applications. We concentrate on the numerical based design, measurement and characterization [16-21] and optimization [22].

The paper is organized as follows. Section 2 gives a description of the manufacturing process for ferroelectret materials. Furthermore, we discuss the principal reasons qualifying these materials for ultrasound transducers. Section 3 deals with the characterization of ferroelectric ultrasound transducers. We investigate single element transducers and transducer arrays by means of numerical simulation as well as measurements. In Section 4 applications are presented which require ultrasound transducers based on ferroelectret materials. A spatially resolved cavitation sensor is discussed here. A conclusion is given in Section 5.

\section{Ferroelectret Material for Transducer Applications}

\section{Manufacturing Process}

Basically, the manufacturing process can be divided into four steps. First, air or grains of sand are blown into polypropylene in the molten state. During the following cooling, spherical cavities with approximately $10 \mu \mathrm{m}$ in diameter develop around the inclusions. In the second step, the modified polypropylene material is extruded in films of $50 \mu \mathrm{m}$ thickness by means of two-dimensional shaking motion and underpressure. Thereby, the spherical cavities are deformed to lenticular voids with $10-100 \mu \mathrm{m}$ 
in diameter and a height of $2-10 \mu$ m (left panel of Fig. 1) $[4,6,23]$. In the third step, the surface roughness of the film is minimized by sealing the top and bottom surface with homogeneous polypropylene. However, in this state the material cannot yet be used as transducer. To activate the material, corona discharge is applied in the last step, whereby electric charges occur at the interface of the lenticular cavities and the polypropylene (right panel of Fig. 1) [24]. Finally, the surface of the film is metalized with an aluminum layer. The piezoelectric properties of the ferroelectret material can further be improved by a second pressure expansion [14]. A wellestablished ferroelectret material for transducers is the so-called EMFi (Electro-Mechanical Film) material (see, e.g., [6], which we used for our applications, too.
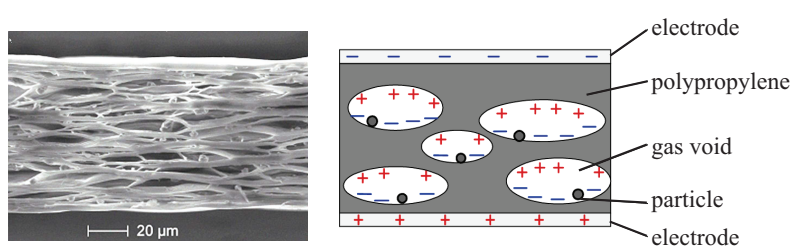

Figure 1: Left: Scanning electron micrograph of the cross section of an EMFi-material [13]. Right: Schematic of the charge distribution inside the film.

\section{Assets and Drawbacks}

In the following, the main assets $\oplus$ and drawbacks $\ominus$ of the EMFi-material for transducer applications are illustrated [4].

$\oplus$ Electro-Mechanical-Coupling: Polyvinylidene Fluoride (PVDF), which is often used for air ultrasound transducers [25] exhibits a piezoelectric strain constant $d_{33}$ in thickness direction of approximately $d_{33}=20 \mathrm{pC} / \mathrm{N}$. Due to the cellular structure, ferroelectret materials offer a high piezoelectric strain constant $d_{33}$ up to $600 \mathrm{pC} / \mathrm{N}$ (see, e.g., [24]). On account of this fact, the EMFi-material is more suitable for air transducers compared to PVDF.

$\oplus$ Acoustic Matching: Due to their cellular structure, ferroelectret materials exhibit a low stiffness yielding a small acoustic impedance [1]. Since the acoustic matching of the transducer material and the surrounding medium is decisive for emitting and receiving acoustic waves, the small acoustic impedance of the EMFi-material is often a major advantage, e.g. for air ultrasound transducers.

$\oplus$ Bandwidth: Because of the mentioned acoustic matching with air, ferroelectret materials provide a large bandwidth. Fig. 2 shows the displacement amplitudes of the EMFi-material (thickness $70 \mu \mathrm{m}$ ) in thickness direction for different excitation voltages as well as frequencies. The displacements have been acquired with a Laser-Scanning-Vibrometer (POLYTEC PSV300) and represent the arithmetic mean over the surface. In the frequency range of $20-$ $180 \mathrm{kHz}$, the displacements stay nearly constant and are in phase. Above the resonance frequency $f_{\mathrm{R}} \approx$ $300 \mathrm{kHz}$ for the thickness mode, remarkable differences in both the magnitudes and the phase angles occur, which can be ascribed to the inhomogeneous structure of the ferroelectret material. However, the behavior in the lower frequency band allows emitting and receiving ultrasound pulses.

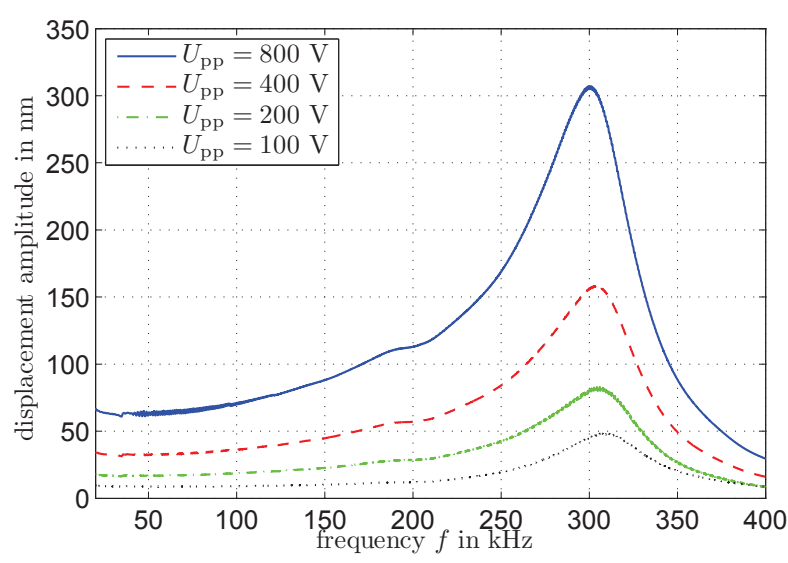

Figure 2: Displacement amplitudes versus frequency of an EMFi-material for different excitation voltages.

$\oplus$ Geometric Dimensions and Mass: Similar to PVDF, ferroelectret materials are thin and flexible films. Thus, it is possible to attach these films to curved surfaces which is often useful in industrial applications. For instance, a spherically curved alignment of the film allows focusing of the emitted pressure waves. Contrary to piezoceramic transducers, the EMFi-material is lightweight and can therefore be used as pressure sensor even in case of tiny as well as lightweight carrier objects.

Temperature Sensitivity: The behavior of ferroelectret materials strongly depends on the ambient temperature. Table 1 contains the resonance frequency $f_{\mathrm{R}}$ with respect of the temperature $\vartheta$ for an EMFi-material. Temperatures higher than $60{ }^{\circ} \mathrm{C}$ cause an irreversible change of the piezoelectric properties. This change can be relocated to higher temperatures by means of fluorocarbonmaterials [26].

Excitation Voltage: As Fig. 2 demonstrates rather high excitation voltages are required to 
Table 1: Resonance Frequency $f_{\mathrm{R}}$ vs. temperature $\vartheta$ for an EMFi-material.

\begin{tabular}{cccccc}
\hline$\vartheta$ in ${ }^{\circ} \mathrm{C}$ & -20 & 0 & 20 & 40 & 60 \\
\hline$f_{\mathrm{R}}$ in $\mathrm{MHz}$ & 0.29 & 0.27 & 0.25 & 0.22 & 0.20 \\
\hline
\end{tabular}

achieve considerable displacements of the ferroelectret material, which is a consequence of the low electromechanical coupling coefficient $k_{33} \approx 0.1$.

\section{Ultrasound Transducers}

\subsection{Single Element Transducers}

Based on the EMFi-material (thickness $70 \mu \mathrm{m} ; d_{33} \approx$ $200 \mathrm{pC} / \mathrm{N}$ ), we assembled different ultrasound transducers. To obtain piezoelectric transducers oscillating in thickness direction, the EMFi-material was fixed at one side to a PCB (Printed Circuit Board) [4, $11,27]$. We used an electrically conductive adhesive to assure electrical connection between the PCB and the bottom electrode of the EMFi-material (see Fig. 3). The top electrode was contacted by thin bond wires. Apart from the transducers consisting of one EMFi layer, we built up stack actuators containing two foils with opposite directions of polarization. Compared to the single layer configuration, stack actuators offer increasing displacements, but also decreasing resonance frequencies for thickness mode. For an ultrasound receiver, both the sensitivity with

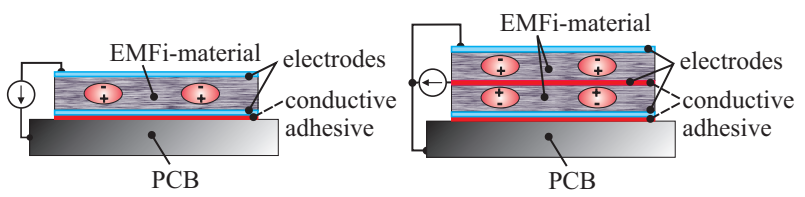

Figure 3: Left panel: Transducer containing one EMFi layer. Right panel: Stack actuator with two EMFi layers.

respect to frequency and the equivalent acoustic noise level are important specifications. Fig. 4 displays the developed receiver, which is based on the EMFi-material. To minimize the size of the receiver, we utilized a multilayer PCB and implemented a low noise preamplifier circuit with SMD (Surface Mounted Device) technology. Fig. 5 shows the sensitivity $B_{\mathrm{R}, \text { Sys }}(f)$ of the receiver (EMFi-material and preamplifier circuit) plotted against the frequency. Two different receiver configurations were investigated, namely a single layered EMFi-material and
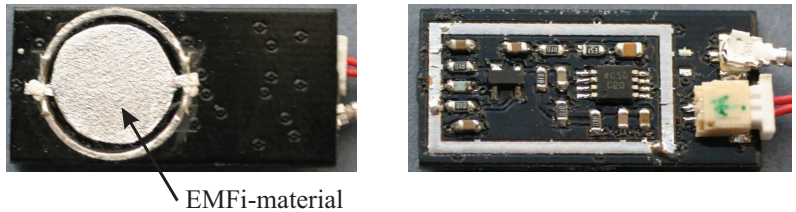

Figure 4: Top and bottom view of the miniaturized receiver, which was implemented with SMD technology.

a stack consisting of two layers. As one can see, the stacked receiver offers mostly a higher value for $B_{\mathrm{R}, \mathrm{Sys}}(f)$. The additional layer increases the mass of the receiver and, therefore, reduces its resonance frequency.

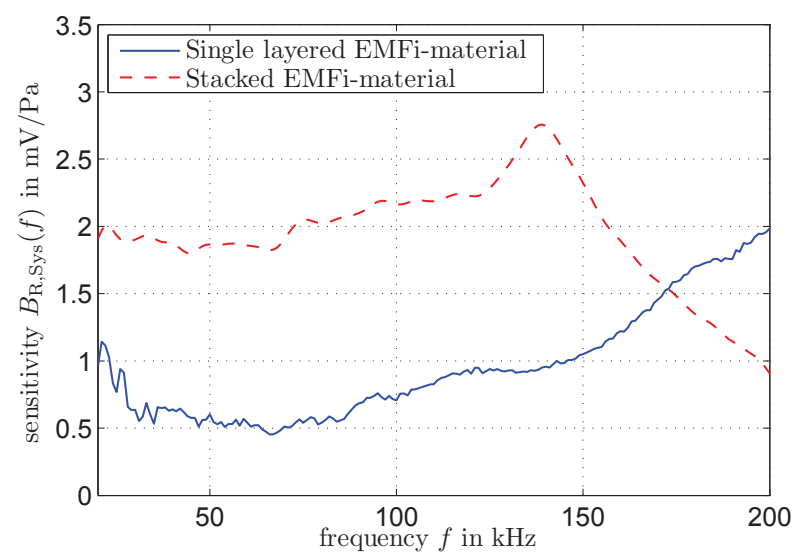

Figure 5: Sensitivity $B_{\mathrm{R}, \text { Sys }}(f)$ of a single layered EMFi receiver and a stacked configuration consisting of two layers versus frequency $f$.

\subsection{Transducer Arrays}

Beside single element transducers, we have also developed transducer arrays based on ferroelectret materials $[4,28]$. There are various technical applications for those transducer arrays. For instance, they can be used for the acquisition of the temporally as well as spatially resolved pressure distributions. Fig. 6 shows an array consisting of 16 circular elements (radius $1.5 \mathrm{~mm}$ ), which we utilized as spatially resolved cavitation sensor (Section 4 ). The elements of the sensor array are arranged in 4 rows and 4 columns. Both the diameter of the elements and the lateral spacings between them were chosen according to the subsequently described application. A squared shaped EMFi-material with an edge length of $20 \mathrm{~mm}$ is fixed onto the bottom electrodes of the 
array elements with a highly viscous and solvent-free adhesive. While the sensor signals are measured at the circular element electrodes, the squared shaped top electrode is the common ground for all sensor elements. In order to protect sensor array and signal lines, an additional coating with $40 \mu \mathrm{m}$ Parylene C layer was used [28]. Thus, the array can be utilized even in case of a harsh environment.

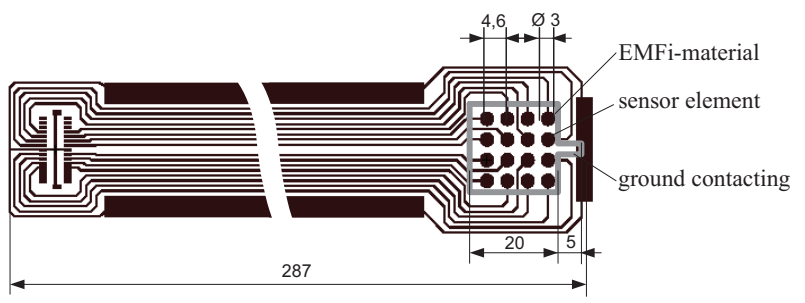

Figure 6: Layout of the sensor elements and the signal lines.

\section{Application: Spatially Resolved Cavitation Sensor}

Ultrasonic cleaning is widely used in industrial applications. The manufacturers are frequently interested in an increased efficiency for the cleaning process. Hence, there is a strong demand for the characterization of cavitation effects, which are the most important mechanism in ultrasonic cleaning. However, common tools (e.g. hydrophones) for measuring those effects disturb the pressure field distribution $[28,29]$. Moreover, a reliable evaluation of the cavitation activity has to be performed directly on the component's surface. That is the reason why we utilized the ferroelectret material as a cavitation sensor [4]. Due to its mechanical flexibility, it can be applied to the components, even in case of curved surfaces. The developed robust sensor array (see Section 3) allows for spatially and temporally resolved measurements of the sound pressure.

In the first step, we verified the applicability of the array as pressure sensor for ultrasound waves propagating in water. For this purpose, we conducted a comparison of hydrophone measurements and the results obtained by the array. Fig. 7 displays both, the pressure distribution acquired with a standard hydrophone (RESON TC 4013) and the electrical output-signal of the sensor array. The measurements were carried out along the symmetry axis of a cylindrically shaped water tank (diameter $140 \mathrm{~mm}$; filling level $200 \mathrm{~mm}$ ). The propagating pressure waves of frequency $f=27.2 \mathrm{kHz}$ were emitted with a piezoelectric stack actuator, which was fixed

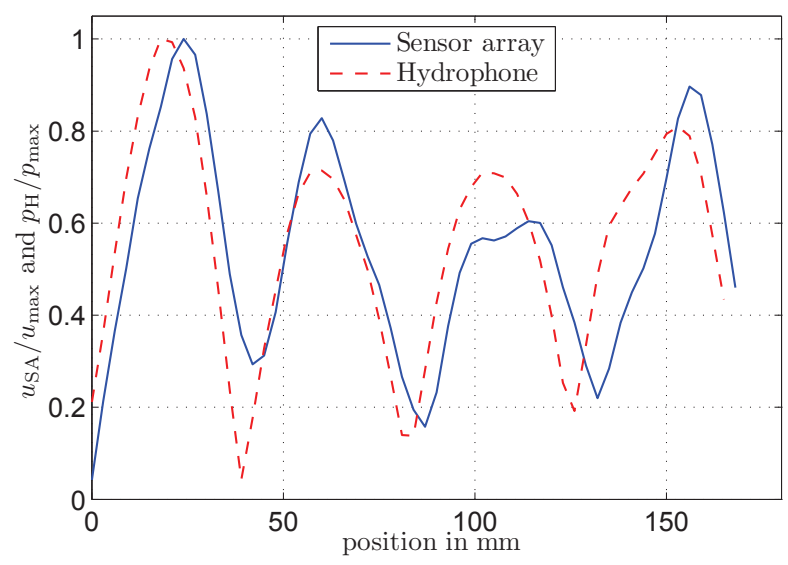

Figure 7: Normalized hydrophone data $p_{\mathrm{H}}$ and normalized electrical output-signal $u_{\mathrm{SA}}$ of the sensor array for the pressure distribution along the symmetry axis of the cylindrically shaped water tank $\left(u_{\max }=0.43 \mathrm{~V}\right.$; $\left.p_{\max }=23.1 \mathrm{kPa}\right)$. The piezoelectric stack actuator was excited with $U_{\mathrm{pp}}=100 \mathrm{~V}$.

at the bottom of the water tank. As the results in Fig. 7 demonstrate, the curve progression of both, the hydrophone data and the array signal are similar. The hydrophone data can be used to calibrate the sensor array. In doing so, the electrical outputsignal of the array can be directly converted to the acoustic pressure.

In the second step, we utilized the array sensor for cavitation measurements. The flexible array was fixed to a cylindrically shaped specimen, which was immersed in the water tank. Fig. 8(a) and (b) depict the spectra resulting from the sensor outputsignal for the transducer excitations $U_{\mathrm{pp}}=100 \mathrm{~V}$ and $U_{\mathrm{pp}}=500 \mathrm{~V}$, respectively. For the transducer voltage $U_{\mathrm{pp}}=100 \mathrm{~V}$, no cavitation was observed inside the water tank. In contrast, a high cavitation activity occurs for the excitation voltage $U_{\mathrm{pp}}=500 \mathrm{~V}$. This is so, because cavitation has an effect on the spectra of the sensor output-signal. While the differences in the fundamental wave (first harmonic; $\left.f_{0}=27.2 \mathrm{kHz}\right)$ and the harmonics $\left(2 f_{0}, 3 f_{0}, \ldots\right)$ are rather small for the two excitations, both, the subharmonic (e.g., $f_{0} / 2$ ) and ultraharmonic (e.g., $3 f_{0} / 2$ ) components arise only for $U_{\mathrm{pp}}=500 \mathrm{~V}$. These results correspond to the dynamic behavior of cavitation bubbles which is reported in literature [30]. The gas concentration (especially the concentration of oxygen) in water strongly affects the cavitation activity [28]. This behavior can also be observed with the aid of the ferroelectret based sensor array. Fig. 9(a) and (c) show the subharmonic $\left(f_{0} / 2\right)$, first harmonic $\left(f_{0}\right)$ and second harmonic $\left(2 f_{0}\right)$ with re- 
(a)

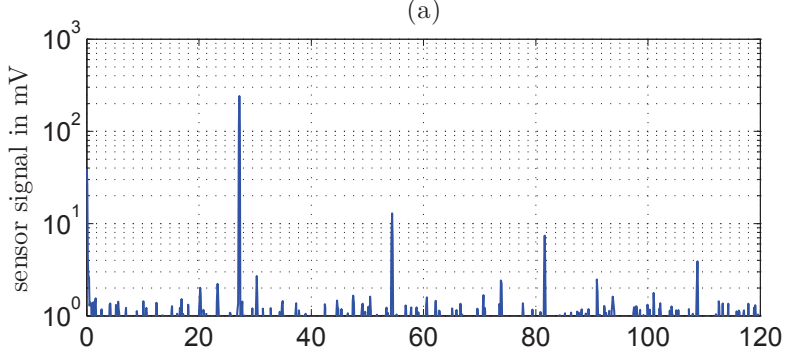

(b)

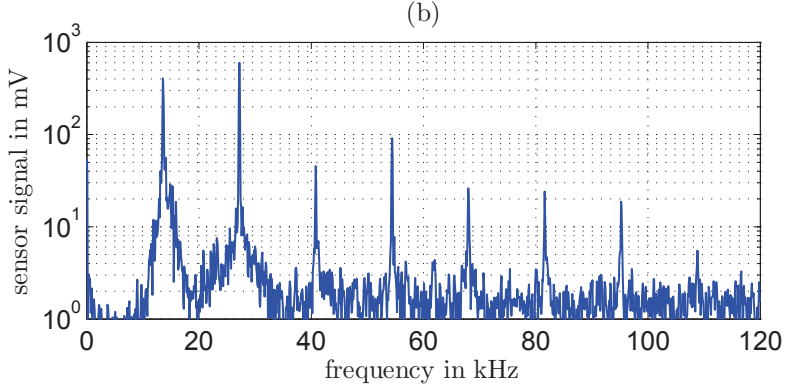

Figure 8: Spectra of the electrical output-signal of the sensor array for the excitation voltages $U_{\mathrm{pp}}=100 \mathrm{~V}$ (a) and $U_{\mathrm{pp}}=500 \mathrm{~V}$ (b) of the piezoelectric stack actuator (excitation frequency $f_{0}=27.2 \mathrm{kHz}$ ).

spect to the excitation voltage $U_{\mathrm{pp}}$ for the oxygen concentration $3.5 \mathrm{mgO}_{2} / \mathrm{l}$ and $7.0 \mathrm{mgO}_{2} / \mathrm{l}$, respectively. It can clearly be seen that the first and second harmonic are barely altered by the excitation voltage. Beside, the subharmonic exhibits rather large changes. However, for an oxygen concentration of $7.0 \mathrm{mgO}_{2} / 1$ the subharmonic remains small even in case of the high excitation voltages. This behavior can also be observed from the test layers (Fig. 9(b) and (d)). While for an oxygen concentration of $7.0 \mathrm{mgO}_{2} / \mathrm{l}$ no ablation occurs, the test layer is extensively damaged in case of $3.5 \mathrm{mgO}_{2} / \mathrm{l}$.

\section{Conclusion}

In this article, we reported on ultrasound transducers, which are made of ferroelectret materials. Due to their cellular structure these materials provide both, a high piezoelectric strain constant and a large bandwidth. However, the low electromechanical coupling coefficient $\left(k_{33} \approx 0.1\right)$ for ferroelectret based transducers requires a fairly high excitation voltage.

In order to demonstrate the applicability of ferroelectret materials (EMFi-materials) for ultrasound applications, we assembled both, single element transducers as well as transducer arrays. A single element ultrasound receiver consisting of one or two layers of EMFi-material and a preamplifier circuit (a)

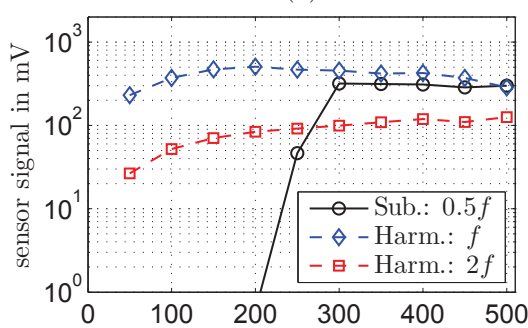

(c)
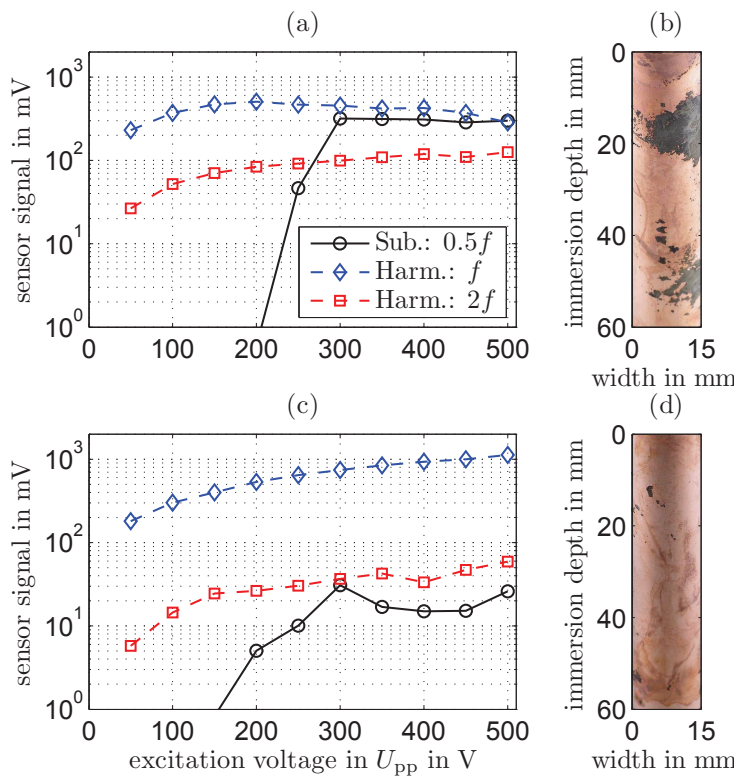

width in $\mathrm{m}$ (d)

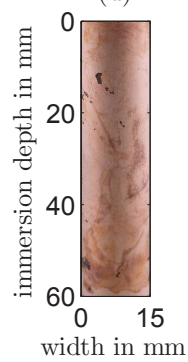

Figure 9: Characteristic spectral components for an oxygen concentration of $3.5 \mathrm{mgO}_{2} / \mathrm{l}$ (a) and $7.0 \mathrm{mgO}_{2} / \mathrm{l}$ (c). The panels (b) and (d) display the corresponding test layers for an excitation voltage of $U_{\mathrm{pp}}=500 \mathrm{~V}$.

was manufactured. Furthermore, a transducer array was set up and characterized by comparing the signal with hydrophone measurements.

The ferroelectret based transducers were utilized in air and liquid applications. Here, the assembled transducer array was utilized as a spatially resolved cavitation sensor. Due to the cellular structure, the EMFi-material can be fixed to specimens even in case of curved surfaces. By means of this sensor, we could identify cavitation activities and their dependency on the oxygen concentration in cleaning systems.

Our future work will be concentrated on further transducer applications of the ferroelectret materials. We want to exploit the outstanding properties of the material for medical applications.

\section{References}

[1] Reinhard Lerch, Gerhard Sessler, and Dietrich Wolf. Technische Akustik. Springer Berlin Heidelberg, 2009.

[2] W. Heywang, K. Lubitz, and W. Wersing. Piezoelectricity: Evolution and future of a technology. Springer Berlin Heidelberg, 2008.

[3] S. J. Rupitsch and B. G. Zagar. Acoustic microscopy technique to precisely locate layer delamination. Instrumentation and Measurement, IEEE Transactions on, 56(4):1429-1434, August 2007. 
[4] S.J. Rupitsch, R. Lerch, J. Strobel, and A. Streicher. Ultrasound transducers based on ferroelectret materials. Dielectrics and Electrical Insulation, IEEE Transactions on, 18(1):69-80, 2011.

[5] G. M. Sessler and J. Hillenbrand. Electromechanical response of cellular electret films. Applied Physics Letters, 75(21):3405 -3407, nov 1999.

[6] M. Paajanen, J. Lekkala, and K. Kirjavainen. Electromechanical film (emfi) - a new multipurpose electret material. Sensors and Actuators A (Physical), 84:95-102, 2000.

[7] R. Lerch. Simulation of piezoelectric devices by twoand three-dimensional finite elements. Ultrasonics, Ferroelectrics and Frequency Control, IEEE Transactions on, 37(3):233-247, May 1990.

[8] R. Kressmann. New piezoelectric polymer for airborne and water-borne sound transducers. Journal of Acoustic Society America, 109(4):1412-1416, 2001.

[9] J.L. Ealo, F. Seco, C. Prieto, A.R. Jimenez, J. Roa, A. Koutsou, and J. Guevara. Customizable field airborne ultrasonic transducers based on electromechanical film. In Ultrasonics Symposium, 2008. IUS 2008. IEEE, pages 879-882, 2-5 2008.

[10] F. Seco, J.L. Ealo, and A.R. Jimenez. Modulation and codification of ultrasonic signals with emfi transducers. In Ultrasonics Symposium (IUS), 2009 IEEE International, pages 1726-1729, 20-23 2009.

[11] A. Streicher. Luftultraschall Sender-EmpfängerSystem für einen künstlichen Fledermauskopf. Ph.D. Thesis, Friedrich-Alexander-University Erlangen-Nuremberg, 2008.

[12] G. Sessler and R. Gerhard-Multhaupt. Electrets, volume 3. Laplacian Press, Morgans Hill, 1999.

[13] G. M. Sessler and J. Hillenbrand. Electromechanical response of cellular electret films. Applied Physics Letters, 75:3405-3407, 1999.

[14] X. Zhang, J. Hillenbrand, and G. M. Sessler. Piezoelectric d33 coefficient of cellular polypropylene subjected to expansion by pressure treatment. Applied Physics Letters, 85(7):1226-1228, Aug. 2004.

[15] S. Bauer. Piezo-, pyro-, and ferroelectrets: Soft transducer materials for electromechanical energy conversion. Dielectrics and Electrical Insulation, IEEE Transactions on, 13(5):953-962, 2006.

[16] S. J. Rupitsch, S. Kindermann, and B. G. Zagar. Estimation of the surface normal velocity of high frequency ultrasound transducers. Ultrasonics, Ferroelectrics, and Frequency Control, IEEE Transactions on, 55(1):225-235, January 2008.

[17] T. Lahmer, M. Kaltenbacher, B. Kaltenbacher, R. Lerch, and E. Leder. Fem-based determination of real and complex elastic, dielectric, and piezoelectric moduli in piezoceramic materials. Ultrasonics, Ferroelectrics and Frequency Control, IEEE Transactions on, 55(2):465-475, Feb. 2008.
[18] S. J. Rupitsch and R. Lerch. Inverse method to estimate material parameters for piezoceramic disc actuators. Applied Physics A: Materials Science \& Processing, 97(4):735-740, December 2009.

[19] S. J. Rupitsch, F. Wolf, A. Sutor, and R. Lerch. Estimation of material parameters for piezoelectric actuators using electrical and mechanical quantities. In Procedings IEEE Ultrasonic Symposium, pages 414417, 2009.

[20] M. Kaltenbacher, B. Kaltenbacher, T. Hegewald, and R. Lerch. Finite element formulation for ferroelectric hysteresis of piezoelectric materials. Journal of Intelligent Material Systems and Structures, 21:773-785, 2010.

[21] A. Sutor, S. J. Rupitsch, and R. Lerch. A preisach based hysteresis model for magnetic and ferroelectric hysteresis. Applied Physics A: Materials Science \& Processing, in press.

[22] F. Wein, M. Kaltenbacher, E Bänsch, G Leugering, and F Schury. Topology optimization of a piezoelectric-mechanical actuator with single- and multiple-frequency excitation. International Journal of Applied Electromagnetics and Mechanics, 30(34):201-221, 2009.

[23] R. Kressmann. Linear and nonlinear piezoelectric response of charged cellular polypropylene. Journal of Applied Physics, 90(7):3489-3496, 2001.

[24] R. Schwödiauer, G. S. Neugschwandtner, K. Schrattbauer, M. Lindner, M. Vieytes, S. Bauer-Gogonea, and S. Bauer. Preparation and characterization of novel piezoelectric and pyroelectric polymer electrets. Dielectrics and Electrical Insulation, IEEE Transactions on, 7(4):578-586, 2000.

[25] R. Lerch and G. M. Sessler. Microphones with rigidly supported piezopolymer membrane. Journal of Acoustic Society America, 67(4):1379-1381, 1980.

[26] X. Zhang, J. Hillenbrand, and G. M. Sessler. Ferroelectrets with improved thermal stability made from fused fluorocarbon layers. Journal of Applied Physics, 101(5):054114 - 054114-8, Mar. 2007.

[27] A. Streicher, M. Kaltenbacher, R. Lerch, and H. Peremans. Broadband emfi ultrasonic transducer for bat research. In Ultrasonics Symposium, 2005 IEEE, volume 3, pages 1629-1632, 18-21 2005.

[28] J. Strobel. Werkzeuge zur Charakterisierung der Kavitation in Ultraschall-Reinigungsbädern. Ph.D. Thesis, Friedrich-Alexander-University ErlangenNuremberg, 2009.

[29] L. Bahr and R. Lerch. Beam profile measurements using light refractive tomography. Ultrasonics, Ferroelectrics and Frequency Control, IEEE Transactions on, 55(2):405-414, Feb. 2008.

[30] F. R. Young. Cavitation. McGraw-Hill Book Company (UK) Ltd., 1989. 